\title{
Hierarchical Two-Layer Distributed Control Architecture for Voltage Regulation in Multiple Microgrids in the Presence of Time-Varying Delays
}

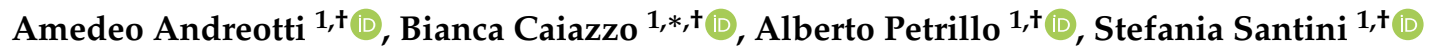 \\ and Alfredo Vaccaro $2,+(1)$ \\ 1 Department of Information Technology and Electrical Engineering (DIETI), University of Naples Federico II, \\ 80125 Naples, Italy; amedeo.andreotti@unina.it (A.A.); alberto.petrillo@unina.it (A.P.); \\ stefania.santini@unina.it (S.S.) \\ 2 Department of Engineering, University of Sannio, 82100 Benevento, Italy; vaccaro@unisannio.it \\ * Correspondence: bianca.caiazzo@unina.it \\ + Authors are in alphabetic order.
}

Received: 3 November 2020; Accepted: 8 December 2020; Published: 9 December 2020

\begin{abstract}
The Multiple Microgrids (MMGs) concept has been identified as a promising solution for the management of large-scale power grids in order to maximize the use of widespread renewable energies sources. However, its deployment in realistic operation scenarios is still an open issue due to the presence of non-ideal and unreliable communication systems that allow each component within the power network to share information about its state. Indeed, due to technological constraints, multiple time-varying communication delays consistently appear during data acquisition and the transmission process and their effects must be considered in the control design phase. To this aim, this paper addresses the voltage regulation control problem for MMGs systems in the presence of time-varying communication delays. To solve this problem, we propose a novel hierarchical two-layer distributed control architecture that accounts for the presence of communication latencies in the information exchange. More specifically, the upper control layer aims at guaranteeing a proper and economical reactive power dispatch among MMGs, while the lower control layer aims at ensuring voltage regulation of all electrical buses within each MG to the desired voltage set-point. By leveraging a proper Driver Generator Nodes Selection Algorithm, we first provide the best choice of generator nodes which, considering the upper layer control goal, speeds up the voltage synchronization process of all the buses within each MG to the voltage set-point computed by the upper-control layer. Then, the lower control layer, on the basis of this desired voltage value, drives the reactive power capability of each smart device within each MG and compensates for possible voltage deviations. Simulation analysis is carried out on the realistic case study of an MMGs system consisting of two identical IEEE 14-bus test systems and the numerical results disclose the effectiveness of the proposed control strategy, as well as its robustness with respect to load fluctuations.
\end{abstract}

Keywords: cluster-oriented coordination; distributed control; multiple microgrids; time-varying delays; IEEE 14-bus test systems

\section{Introduction}

The increasing use of Renewable Energy Sources (RESs), Distributed Generators (DGs) and storage systems is imperative for the introduction of Microgrids (MGs) in actual power distribution networks because of the many benefits it could lead to in terms of reductions in power losses and electrical system performance improvements. In general, an MG is a small distribution power system 
consisting of DGs, energy storage systems (ESSs) and local loads [1] collectively handled to increase the hosting capacity of RESs and to improve energy security, thus offering flexibility service to the grid.

There exist two MG operation modes-stand-alone and grid connected mode [2]. In grid-connected mode, the MG is tied to the main grid trough the Point of Common Coupling (PCC), allowing power exchange from or to the main grid and voltage/frequency regulation according to utility specification [3]. When the MG opens the PCC, it operates in islanded mode, i.e., the MG works independently of the main grid, trying to maintain its own voltage and frequency to the reference values, while guaranteeing optimal power sharing among DGs and loads [4]. Modeling, stability and control of an MG are hot topics in the research field and many control techniques have been developed to address the main issues within an MG such as voltage fluctuations, flickers and instability due to the variable nature of DG units and the accurate reactive power sharing $[5,6]$. Since these future power grids can be restructured as cyber-physical systems, whose components not only deal with power flow management but also with data transmission to ensure a distributed control capability [7], most of the existing works in the technical literature leverage Multi-Agent System (MAS) framework in order to model the resulting power network. Thus, a self-organized architecture is obtained, which allows the cooperative and adaptive control of all intelligent electrical components so to achieve both local and global objective functions [8-11], even for communication time-delays and cyber networks with uncertain communication links [12].

However, since many DGs in an islanded MG system increase the investment cost and complicate the network topology [13], in order to allow the maximum utilization of RESs while suppressing stressing and aging of the components, multiple MGs clusters (MMGs) are identified as the future trend of the smart distribution grids due to the reliability and the availability they can guarantee [14]. An MMG is a set of MGs geographically close to each other, which are able to share information about their power management and are considered together as a unique entity to improve the operational stability and economic benefits of each MG [15], while enhancing the resilience of the whole power system to external disturbances [16]. The technical literature classifies MMGs into three categories: (1) low voltage (LV), where MGs are interconnected through LV tie lines; (2) medium voltage (MV), where MGs are connected via MV feeders; (3) LV MGs interconnected through a MV feeder and distribution transformers [17]. The main idea of using the MMGs paradigm is to form a Smart MGs Network (SMGN) to maximize the utilization of RESs. Indeed, this concept allows for (i) the sharing of reserves in critical conditions to reduce the risk of system collapse and minimize the emergency load shedding requirement; (ii) guaranteeing of the economical dispatch in the whole power network; (iii) sharing of storage and ancillary functions [18]. For instance, when some MGs are not able to meet the required power consumption, other MGs, having stored energy, could help them by sharing its sources [19].

Although the technical literature significantly addresses stability and control challenges of an MG (see the surveys $[20,21]$ and references therein), to date, there are few studies focusing on a multiple MGs cluster in the SMGN context [19,22]. The coordination of the different MGs incorporated into the SMGN is a crucial technical challenge since their interconnection may lead to the instability of the overall power system. More specifically, one of the most critical points for MMGs is to select a suitable overlay communication topology to describe the optimal interconnection among different MGs with RESs [19]. To solve the problem, Che et al. [18] propose a probabilistic minimal cut-set-based iterative methodology to investigate the reliability and the redundancy. Looking at a single MG as a cluster of different entities, Cortes et al. [23] suggest an iterative procedure for the optimal design of an MG topology through partitioning, integer programming and performance index methods, while, based on demand response program, Ajoulabadi et al. [24] present an optimal reconfiguration of multiple MGs in order to improve scheduling flexibility, thus meeting the best scheduling objectives.

Besides the appropriate selection of the MGs network topology, another fundamental issue in MMGs is attaining the optimal power dispatch among the different MGs, i.e., the so-called Energy Management (EM) problem that deals with the coordination of DG units within each MG, as well 
as the power trading between the main grid and MGs. To address this issue, different approaches have been proposed for the coordination of the MMGs system [25], namely master-slave control [26], peer-to-peer control [27] and hierarchical control [28]. Leveraging this latter framework, a hierarchical decentralized SoS architecture for the energy management of an MMGs system has been proposed in [15] by formulating a bi-level optimization problem and considering the RESs uncertainty, while [29] exploits a smart transformer-based approach. Again, a hierarchical two-layer control architecture for a cluster of islanded MGs sharing information via intermittent communications has been proposed in $[22,30]$.

Although the solutions proposed herein allow for the coordination control problem for MMGs to be effectively and reliably solved, their design leverages the restrictive assumption of an ideal and always reliable communication among the different electrical components constituting the whole power grid system. However, when considering a wireless communication network, based on, for example the IEEE 802.11 protocol, each communication link that connects a pair of agents is affected by a different variable time-delay whose value depends on actual conditions, or possible impairments, of the communication channel [11]. It follows that the hypothesis commonly made in the technical literature of an ideal communication network in the MMGs may be unrealistic. Hence, the control architecture deployment in realistic operation scenarios is still an open problem, which warrants further investigation $[17,22]$.

To overcome this limitation and solve the problem of determining the optimal power dispatch in practical MMGs systems, we propose a novel cooperative cluster-oriented hierarchical control architecture that accounts for the presence of communication time-varying delays. Exploiting the Multi-Agent System (MAS) mathematical framework, the proposed control architecture is based on a network of cooperative smart controllers (called nodes), each one regulating the voltage magnitude of a specific bus within each MG cluster constituting the whole MMGs system. All nodes/controllers are able to share information about their states with their neighbors (within their communication range) so that the control actions can be cooperatively computed by embedding within the online decision-making process not only information coming from local sensing, but the delayed network information about the surroundings. More specifically, the proposed control solution consists of two cyber layers, namely the upper and lower control layers. The upper control layer aims at guaranteeing a suitable and economical reactive power dispatch among multiple MGs. This can be done by controlling only a specific subset of the DGs within each MG, i.e., the driver generator nodes, via a cooperative control action that, based on the knowledge of the rated voltage value to be imposed to the whole MMGs and the neighboring delayed information shared with other drivers belonging to other MGs through the inter-cluster communication network, determines the voltage reference value for each MG. The driver generator nodes set, as well as the inter-cluster communication network topology, is computed by leveraging the Master Stability Function (MSF) approach [31,32] which determines the best choice of these nodes for speeding up the voltage synchronization process of all buses within each MG to the safe voltage set-point computed by the upper-control layer. Conversely, based on this latter point, the lower control layer aims at ensuring voltage regulation of all $P V / P Q$ buses within each MG to the desired voltage set-point. This is done via a cooperative control action that, by exploiting delayed state information shared among the smart devices within the single MG via the intra-cluster communication network, drives the reactive power generation capability of each smart device and compensates for possible voltage deviations.

To prove the effectiveness of the proposed cluster-oriented cooperative control strategy, we consider an MMGs system consisting of two identical MGs, i.e., the IEEE 14-bus test system. The results confirm the ability of the proposed solution to guarantee the two-fold voltage regulation of the MMGs system, despite the presence of communication time-varying delays and/or possible load variations.

The paper is organized as follows. Section 2 describes the voltage control problem for MMGs systems, as well as the dynamics of the cooperative smart controllers network that was exploited 
for its control. The Cluster-Oriented Cooperative control strategy, together with Driver Generator Nodes Selection Algorithm based on MSF formalism, is detailed in Section 3. Section 4 discloses the effectiveness and robustness of the proposed approach for the exemplar MMGs system consisting of two IEEE 14-bus test system. Finally, conclusions are drawn in Section 5.

\section{Multiple MicroGrids Modeling}

Consider an MMGs system consisting of $M$ MGs, labeled as $M G_{1}, \ldots, M G_{k}, \ldots, M G_{M}$ as in Figure 1.

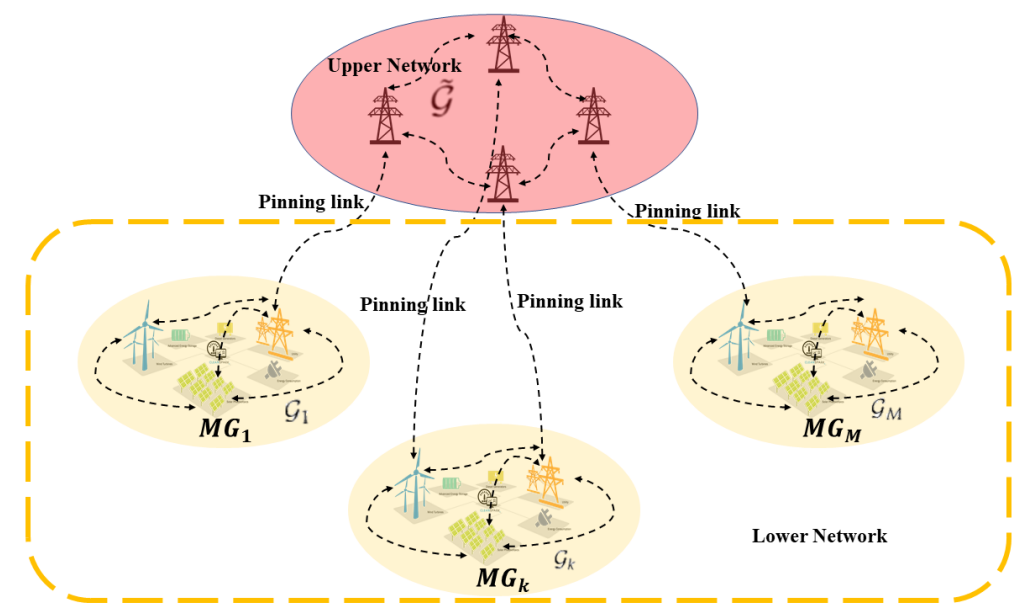

Figure 1. Microgrid (MG) cluster supported by a double-layer communication network: the upper network is highlighted in red and the lower network is highlighted in yellow.

Each power grid $M G_{k}\left(\forall k \in \mathcal{I}_{M}\right)$ consists of $N_{c}^{k}$ capacitor banks, $N_{g}^{k}$ distributed generators and $N^{k}=N_{g}^{k}+N_{c}^{k}$ buses managed by $N^{k}$ cooperative smart controllers. Leveraging the Cluster-Oriented Cooperative Control strategy [28] and Distributed Hierarchical Cooperative (DHC) framework [22], each power grid $M G_{k}$ is able to share information via wireless inter-cluster communication networks with its neighbors $M G_{k^{\prime}}$, being $k^{\prime} \in \mathcal{I}_{M}$ with $k \neq k^{\prime}$. Moreover, each smart device $j$ within the single $M G_{k}$ (being $j \in \mathcal{I}_{N^{k}}$ ) can communicate via an intra-cluster wireless communication network with its neighboring smart devices $q$, being $q \in \mathcal{I}_{N^{k}}$ with $q \neq j$. The intra-cluster communication network structure corresponds to the information exchange topology among the $N^{k}$ smart controllers within the $M G_{k}$. The inter-cluster communication, instead, is only enabled for $n_{k}$ smart devices within each $M G_{k}$, associated to some specific generators buses and named drivers/pinners. This inter-cluster communication network structure, indicated with $\tilde{\mathcal{G}}$, defines the information flow shared among the different $l$ MMGs pinners, being $l \in \mathcal{I}^{\text {pin }}$.

This decomposition of the MMGs into two cyber layers, i.e., the upper and the lower ones, enables a twofold voltage regulation control, as well as an optimal power management of the entire MMGs system [22]. In particular, supposing that the pinners of each MG can access the voltage-rated value $V^{\text {rated }}$ to be imposed to the whole MMGs system, the upper inter-cluster layer aims at guaranteeing a correct and economical reactive power dispatch among multiple MGs by computing the reference voltage set value, i.e., $V_{k}^{\text {nom }}$, to be sent to each pinner $s \in \mathcal{I}_{n_{k}}^{\text {pin }}$ (being $k \in \mathcal{I}_{M}$ ). Conversely, the lower intra-cluster layer aims at ensuring a faster voltage synchronization process of all $\mathrm{PV} / \mathrm{PQ}$ buses within the $M G_{k}$ to the voltage set-point $V_{k}^{\text {nom }}$ by controlling the reactive power generation capability of each $M G_{k}$ bus to compensate for voltage deviations. Note that, for each $M G_{k}$, the optimal identification of the pinner generator nodes, i.e., $n_{k}$, as well as their best positions in the grid are identified according to the MSF formalism within Pinning Control Theory, which proves that the most influential nodes are highly dependent on the network structure [31,33,34]. 


\subsection{Double-Layer Communication Network}

The double-layer communication network describing the information exchange among the smart controllers within the MMGs in the cyber-physical space can be modeled according to graph theory.

Regarding the intra-cluster network for each $M G_{k}\left(k \in \mathcal{I}_{M}\right)$, the communication topology of the $N_{c}^{k}$ cooperative smart device that controls the capacitor bank buses can be described as a directed graph $\mathcal{G}_{N_{c}^{k}}=\left\{\mathcal{V}_{N_{c}^{k}}, \mathcal{E}_{N_{c}^{k}}, \mathcal{A}_{N_{c}^{k}}\right\}$ characterized by the set of nodes $\mathcal{V}_{N_{c}^{k}}=\left\{1, \ldots, N_{c}^{k}\right\}$ and the set of edges $\mathcal{E}_{N_{c}^{k}} \subseteq \mathcal{V}_{N_{c}^{k}} \times \mathcal{V}_{N_{c}^{k}}$. The associated adjacency matrix with non-negative elements is $\mathcal{A}_{N_{c}^{k}}=\left[\alpha_{p \rho}^{k}\right]_{N_{c}^{k} \times N_{c}^{k^{\prime}}}$ being $p, \rho \in \mathcal{I}_{N_{c}^{k}}$ with $p \neq \rho$. We assume $\alpha_{p \rho}^{k}=1$ in the presence of a communication link from the device $p$ to device $\rho$, otherwise $\alpha_{p \rho}^{k}=0$. Moreover, $\alpha_{p p}^{k}=0$, i.e., self-edges $(p, p)^{k}$ are not allowed. The presence/absence of connections among the $N_{c}^{k}$ cooperative smart controller and the $v^{k}=N_{g}^{k}-n_{k}$ smart controller for the non-pinner generation buses is instead described by the overall graph $\mathcal{G}_{k}=\left\{\mathcal{V}_{k}, \mathcal{E}_{k}, \mathcal{A}_{k}\right\}$, where $\mathcal{V}_{k}=\left\{1,2, \ldots, N_{c}^{k}, N_{c}^{k}+1, \ldots, \epsilon^{k}\right\}$ is the set of the $\epsilon^{k}=N_{c}^{k}+v^{k}$ smart controllers, while $\mathcal{E}_{k} \subseteq \mathcal{V}_{k} \times \mathcal{V}_{k}$ represents the set of edges describing the communication links. In this way, the communication structure can be described by the adjacency matrix $\mathcal{A}_{k}=\left[a_{i p}^{k}\right] \in \mathbb{R}^{\epsilon^{k} \times \epsilon^{k}}$ whose generic element $a_{i p}^{k}=1$ if there is a link among the smart devices $i$ and $p$, being $i, p \in \mathcal{I}_{k}^{\text {lower }}$, $a_{i p}^{k}=0$ otherwise. To model the communication of each smart device $j \in \mathcal{I}_{k}^{\text {lower }}$ with the $n_{k}$ pinners, we introduce the leader-adjacency matrix $\mathcal{B}_{k}=\operatorname{diag}\left\{\mathrm{b}_{\mathrm{js}}^{\mathrm{k}}\right\} \in \mathbb{R}^{\epsilon^{k} \times \epsilon^{k}}$, whose value is $b_{j s}^{k}=1$ if the $j$-th bus controller receives information from $s$-th driver generator bus (being $s \in \mathcal{I}_{n_{k}}^{p i n}$ ), otherwise $b_{j s}^{k}=0$.

Similarly, the upper inter-cluster cyber-network is described via the digraph $\tilde{\mathcal{G}}=\{\tilde{\mathcal{V}}, \tilde{\mathcal{E}}, \tilde{\mathcal{A}}\}$ with virtual node set $\tilde{\mathcal{V}}=\left\{\mathcal{V}_{1}^{\text {pin }}, \ldots, \mathcal{V}_{M}^{\text {pin }}\right\}$ being $\mathcal{V}_{k}^{\text {pin }}$ the set of driver generator nodes for the $k$-th MG with cardinality $\operatorname{card}\left(\mathcal{V}_{k}^{\text {pin }}\right)=n_{k}<N_{g}^{k} . \tilde{\mathcal{E}} \subseteq \tilde{\mathcal{V}} \times \tilde{\mathcal{V}}$ represents the set of cyber communication links, while the adjacency matrix $\tilde{\mathcal{A}}=\left[a_{s l}\right] \in \mathbb{R}^{n \times n}$ (with $n=\sum_{k=1}^{M} n_{k}$ ) is such that $a_{s l}=1$ if there is a link from pinner generator $s \in \mathcal{I}^{\text {pin }}$ to pinner generator $l \in \mathcal{I}^{\text {pin }}$, with $s \neq l$.

Finally note that, since, in practice, communication networks are commonly affected by latency in the information delivery due to the current conditions of the communication infrastructure, an unknown heterogeneous time-varying delay $\tau_{q j}(t)$ (being $q$ and $j$ generic electrical nodes) can be associated to each direct edge. Although delays are time-varying, they are usually bounded during the normal operating conditions of technological communication networks, hence $\tau_{q j}(t) \in\left[0, \tau^{\star}\right]$ and $\dot{\tau}(t) \leq \mu$ with $\mu \in[0,1)[35]$.

\subsection{Cooperative Smart Agents Dynamics}

The dynamics of each smart device $p$ within the $k$-th MG and associated to the $p$-th capacitor bank bus is described $\forall p \in \mathcal{I}_{N_{c}^{k}}$ and $\forall k \in \mathcal{I}_{M}$ by the following dynamical system [11]:

$$
\dot{Q}_{k, p}=u_{k, p}\left(t, \tau_{p j}^{k}(t), \tau_{p s}^{k}(t)\right)
$$

where $Q_{k, p}[p . u$.$] is the reactive power of the p$-th capacitor bank within the $k$-th MG; $u_{k, p}\left(t, \tau_{p j}^{k}(t), \tau_{p s}^{k}(t)\right)$ is the distributed cooperative control protocol that, by exploiting the local measurement and the ones shared via the intra-cluster communication network, need to be designed in order to drive the reactive power of the capacitor bank bus, as well as its voltage magnitude; $\tau_{p j}^{k}(t)\left(p \in \mathcal{I}_{N_{c}^{k}}, j \in \mathcal{I}_{k}^{\text {lower }}, p \neq j\right)$ and $\tau_{p s}^{k}(t)\left(s \in \mathcal{I}_{n_{k}}^{\text {pin }}\right)$ are the heterogeneous time-varying communication delays affecting the communication among each pair of electrical nodes. Their actual values, at a given time-instant, depend on the current availability of the wireless channels themselves.

Note that, since the capacitor bank is composed of PQ buses and its voltage magnitude can be regulated by imposing a proper reactive power variation, (1) well represents this kind of phenomena and allows for the reactive power of the capacitor bank to be adapted to guarantee voltage regulation (see $[11,36]$ and references therein). 
The dynamics of the $i$-th smart device controlling the non-pinner generators nodes within $\mathrm{MG}_{k}$ $\left(\forall k \in \mathcal{I}_{M}\right)$ is described $\forall i \in \mathcal{I}_{k}^{\text {non-pin }}$ as [11]:

$$
\dot{V}_{k, i}=u_{k, i}\left(t, \tau_{i j}^{k}(t), \tau_{i s}^{k}(t)\right)
$$

where $V_{k, i}[p . u$. $]$ represents the voltage magnitude of the $i$-th non-pinner generator bus within the $k$-th MG; $u_{k, i}\left(t, \tau_{i j}^{k}(t), \tau_{i s}^{k}(t)\right)$ is the control action to be designed, exploiting both the local measurement and the ones shared via the intra-cluster communication network in order to synchronize the voltage of the $i$-th non pinner generator bus to the desired nominal value $V_{k, s}^{n o m} ; \tau_{i j}^{k}(t)$ is the heterogeneous time-varying delay affecting the information shared between the non-pinner DG $i$ (being $i \in \mathcal{I}_{k}^{\text {non-pin }}$ ) and the smart device $j$ (being $j \in \mathcal{I}_{k}^{\text {lower }}$ ), while $\tau_{i s}^{k}(t)$ is the communication delay affecting the exchanging data between non-pinner $i$-DG and $s$-th DG pinner $\left(s \in \mathcal{I}_{n_{k}}^{\text {pin }}\right)$ within the $k$-th MG.

The behaviour of each smart device controlling the driver generator bus $s\left(\forall s \in \mathcal{I}_{n_{k}}^{p i n}\right)$ within $M G_{k}$ $\left(\forall k \in \mathcal{I}_{M}\right)$ is described by the following dynamical system:

$$
\dot{V}_{k, s}^{\text {nom }}=u_{k, s}\left(t, V^{\text {rated }}, \tau_{l s}(t)\right),
$$

where $V_{k, s}^{n o m}[p . u$. $]$ is the voltage magnitude of the $s$-th driver generator within the $k$-th MG; $V^{\text {rated }}[p . u$. $]$ is the known voltage magnitude set-point to be imposed to the whole MMGs system; $u_{k, s}\left(t, V^{\text {rated }}, \tau_{l s}(t)\right)$ is the cooperative control action which, leveraging both local measurements and the information shared with the other pinners $l\left(\forall l \in \mathcal{I}^{\text {pin }}\right)$ via the inter-cluster communication network $\tilde{\mathcal{G}}$, computes the voltage nominal reference value $V_{k, s}^{\text {nom }}=V_{k}^{\text {nom }}$ to be imposed to the $k$-th MG based on both the knowledge of $V^{\text {rated }}$ and the reactive power mismatch among the MGs; $\tau_{l s}(t)$ is the heterogeneous time-varying delay affecting the data exchange between the pinner generator node $l$ within $\mathrm{MG}_{k^{\prime}}$ and the pinner $s$ within $M G_{k}$.

We remark that the $s$-th pinner generator node, $\forall s \in \mathcal{I}_{n_{k}}^{\text {pin }}$, acts as a leader for the whole $\mathrm{MG}_{k}$ in the lower control layer by imposing the reference voltage magnitude $V_{k}^{n o m}$ for both the capacitor bank and non-pinner generator nodes.

\section{Design of Cluster-Oriented Cooperative Control Strategy}

In what follows, we address the two-layer voltage regulation control problem and the related power management of the MMGs system. The design of the cooperative controllers starts with the appropriate selection of the $n_{k}$ driver generator nodes within each $M G_{k}, \forall k \in \mathcal{I}_{M}$. The pinner selection algorithm leverages the MSF approach [31] and guarantees the choice of the optimal nodes for speeding up the voltage synchronization process of all buses within each MG to a safe voltage set-point via the double-layer cooperative control strategy. Once the driver nodes set $\mathcal{I}_{n_{k}}^{\text {pin }}$ is defined for each $M G_{k}$, the upper cooperative control layer, leveraging the inter-cluster cyber communication topology and the knowledge of $V^{\text {rated }}$, computes the nominal reference value $V_{k}^{\text {nom }}$ to be sent to each $M G_{k}$. On the basis of this latter point, the lower cooperative control layer, exploiting the intra-cluster cyber communication topology, guarantees that each electrical node within the $M G_{k}$ reaches this reference voltage value despite the presence of communication delays.

\subsection{Driver Generator Nodes Selection}

Here, we present the selection algorithm based on MSF that we leverage to find the best driver generator nodes [31,32]. It provides a suitable criteria to find the most influential nodes set in each $M G_{k}, k \in \mathcal{I}_{M}$, i.e., $\mathcal{I}_{n_{k}}^{\text {pin }}$, and hence, the overall set $\mathcal{I}^{\text {pin }}=\bigcup_{k=1}^{M} \mathcal{I}_{n_{k}}^{\text {pin }}$ involved in the upper inter-cluster control layer, needed to perform an accurate economical power allocation within the whole MMGs system. Specifically, for each $M G_{k}$, the algorithm selects within the set $\mathcal{I}_{N_{g}^{k}}$, the $n_{k}<N_{g}^{k}$ driver generator nodes. This is performed through the evaluation of the matrix $C$ structure. The latter is 
obtained by considering all the possible configurations for the Laplacian matrix of the intra-cluster communication network, i.e., $L=\left[l_{i j}\right]=D-A$, with $A$ being the corresponding adjacency matrix and $D$ the in-dregree matrix, augmented with the driver generator nodes on the main diagonal as

$$
C=\left\{c_{i j}\right\}=\left[\begin{array}{cccc}
l_{11}+\beta_{1} & l_{12} & \ldots & l_{1 N^{k}} \\
l_{21} & l_{22}+\beta_{2} & \ldots & l_{2 N^{k}} \\
\vdots & \vdots & \vdots & \vdots \\
l_{N^{k}} & l_{N^{k}} & \ldots & l_{N^{k} N^{k}}+\beta_{N^{k}}
\end{array}\right]
$$

where $\beta_{i}=1$ if the generic node $i$ is a driver generator node, 0 otherwise, $\forall i=1, \cdots, N^{k}$. Iteratively, for each possible matrix $C$, the algorithm computes the eigenratio $R$, defined as

$$
R=\frac{\lambda_{N}}{\lambda_{1}}
$$

with $\lambda_{N}$ and $\lambda_{1}$ being the biggest and smallest eigenvalues of the matrix $C$, respectively. Then, among all the possible driver node configurations, the algorithm selects the ones such that $R<\bar{R}$, with $\bar{R}$ being a positive threshold that guarantees that the Master Stability Function is negative [32]. Finally, the algorithm solves an optimization problem whose solution returns the best driver node configuration, indicated as $C^{\star}$ with the minimum eigenratio $R^{\star}$.

This optimization problem can, hence, be formulated as

$$
\begin{gathered}
\min _{\beta_{i}} R(\beta)=\frac{\lambda_{N}(\beta)}{\lambda_{1}(\beta)}<\bar{R} \\
\text { s.t. } \sum_{j=1}^{N^{k}} \beta_{j}=n_{k}
\end{gathered}
$$

where $\beta=\left[\beta_{1}, \beta_{2}, \ldots, \beta_{N^{k}}\right]$ is the optimization variable representing the number and the position of the driver nodes as well as $n_{K}$ the total number of them.

The selection algorithm for the driver generator nodes set $\mathcal{I}_{n_{k}}^{\text {pin }}$ for each $\mathrm{MG}_{k}$ is summarized in Algorithm 1.

\subsection{Inter-Cluster Cooperative Control Strategy Design}

The upper inter-cluster cooperative control layer aims at adjusting the power flow among the $M$ MGs to achieve the following output reactive power balance:

$$
\lim _{t \rightarrow \infty}\left|Q_{k^{\prime}}(t)-Q_{k}(t)\right|=0
$$

where $Q_{k}(t)\left[p . u\right.$.] and $Q_{k^{\prime}}(t)[p . u$.$] are the total reactive power outputs of \mathrm{MG}_{k}$ and $\mathrm{MG}_{k^{\prime}}^{\prime}$, respectively, $\forall k, k^{\prime} \in \mathcal{I}_{M}$ with $k \neq k^{\prime}$. Considering that the inter-cluster communication is enabled only for the $n$ driver generator nodes within the MMGs system, the control goal as in (7) can be recast as the following cooperative consensus problem:

$$
\lim _{t \rightarrow \infty}\left|Q_{k^{\prime}, l}(t)-Q_{k, s}(t)\right|=0
$$

where $Q_{k^{\prime}, l}(t)[p . u$.$] and Q_{k, s}(t)[p . u$.$] are the reactive power outputs of pinner generator nodes l$ and $s$, respectively, with $l \in \mathcal{I}_{n_{k^{\prime}}}^{\text {pin }}, s \in \mathcal{I}_{n_{k}}^{\text {pin }}$ being $\forall k, k^{\prime} \in \mathcal{I}_{M} k \neq k^{\prime}$. Since each pinner generator node $s$ within $M G_{k}$ (i.e., $s \in \mathcal{I}_{n_{k}}^{\text {pin }}, k \in \mathcal{I}_{M}$ ) knows the voltage rated value to be imposed on the whole MMGs 
system, i.e., $V^{\text {rated }}$ ), in order to achieve (8), we design the cooperative control action for the pinner $s$ within each $M G_{k}$ in (3), as

$$
u_{k, s}\left(t, V^{\text {rated }}, \tau_{l s}(t)\right)=V^{\text {rated }}+k_{Q}\left(\sum_{l \in \mathcal{I}^{\text {pin }}} a_{s l}\left(Q_{k^{\prime}, l}\left(t-\tau_{l s}(t)\right)-Q_{k, s}\left(t-\tau_{l s}(t)\right)\right)\right)
$$

where $k_{Q}$ is the control gain; $a_{s l}$ models the upper inter-cluster cyber-network emerging from the presence/absence of communication link between the $s$-th and the $l$-th pinner generator of $\mathrm{MG}_{k}$ and $\mathrm{MG}_{k}^{\prime}$, respectively; $\tau_{l s}(t)$ is the heterogeneous time-varying delay depending on the specific communication link, with $s \in \mathcal{I}_{n_{k}}$ and $l \in \mathcal{I}_{n_{k^{\prime}}}$. Indeed, since smart devices share information through a wireless communication network, there can be a time delay in the communication among devices as a result of the non-ideality of communication channels and the limited bandwidth. The above-mentioned considerations lead to the need of running the controller based on outdated information and of using the time stamp inserted into messages to correctly correlate the information.

Finally, note that the cooperative control strategy in (9) also generates the voltage nominal reference value $V_{k, s}^{n o m}=V_{k}^{\text {nom }}$ to be imposed on all the $P V / P Q$ buses within each $k$-MG via the lower intra-cluster control layer.

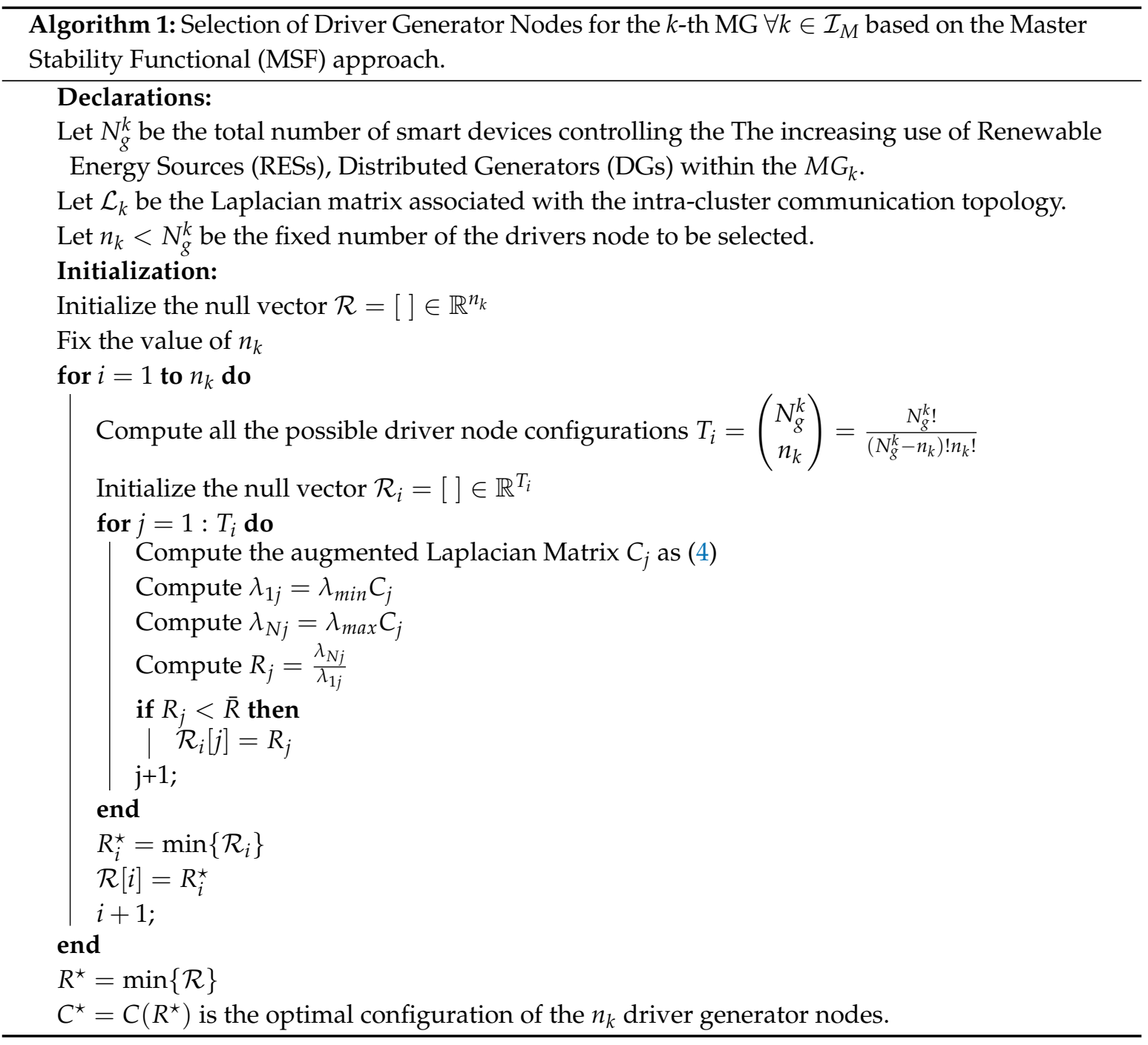




\subsection{Intra-Cluster Cooperative Control Strategy Design}

The intra-cluster voltage regulation control problem for each $M G_{k}$ requires the achievement of the following control goals $\forall k \in \mathcal{I}_{M}$ :

1. To design a fully distributed cooperative controller $u_{k, i}\left(t, \tau_{i j}^{k}(t), \tau_{i s}^{k}(t)\right)$ in (2), which, based on local measurements and outdated networked information, regulates the voltage magnitude $V_{k, i}[p . u$. $]$ of the $i$-th non-pinner generator bus within $M G_{k}\left(i \in \mathcal{I}_{k}^{\text {non-pin }}\right)$ in order to reach and maintain the desired reference voltage value $V_{k, s}^{n o m}[p . u].\left(\forall s \in \mathcal{I}_{n_{k}}\right)$ computed by the upper control level, i.e.,:

$$
\begin{aligned}
& \lim _{t \rightarrow \infty}\left|V_{k, i}\left(t-\tau_{i j}^{k}(t)\right)-V_{k, j}\left(t-\tau_{i j}^{k}(t)\right)\right|=0, \\
& \lim _{t \rightarrow \infty}\left|V_{k, i}\left(t-\tau_{i s}^{k}(t)\right)-V_{k, s}^{n o m}\left(t-\tau_{i s}^{k}(t)\right)\right|=0,
\end{aligned}
$$

where $V_{k, j}[p . u$.$] is the voltage magnitude of the generic bus j \in \mathcal{I}_{k}^{\text {lower }}$ with $i \neq j$ and $V_{k, s}^{\text {nom }}=$ $V_{k}^{n o m}[p . u$.$] is the nominal voltage magnitude of the s$-th driver generator node, $\forall s \in \mathcal{I}_{n_{k}}^{p i n}$.

2. To design a fully distributed cooperative control protocol $u_{k, p}\left(t, \tau_{p j}^{k}(t), \tau_{p s}^{k}(t)\right)$ in (1), which opportunely drives the reactive power of the capacitor bank $p\left(p \in \mathcal{I}_{N_{c}^{k}}\right)$ by updating its voltage magnitude $V_{k, p}\left[p . u\right.$.] until it reaches the desired reference behavior as imposed by the $N_{g}^{k}$ generators within the $M G_{k}$; i.e:

$$
\begin{aligned}
& \lim _{t \rightarrow \infty}\left|V_{k, p}\left(t-\tau_{p j}^{k}(t)\right)-V_{k, j}\left(t-\tau_{p j}^{k}(t)\right)\right|=0, \\
& \lim _{t \rightarrow \infty}\left|V_{k, p}\left(t-\tau_{p s}^{k}(t)\right)-V_{k, s}^{n o m}\left(t-\tau_{p s}^{k}(t)\right)\right|=0,
\end{aligned}
$$

where $V_{k, p}[p . u$. $]$ is the voltage magnitude of the generic bus $p \in \mathcal{I}_{k}^{\text {lower }}$ with $p \neq j$ and $V_{k, s}^{\text {nom }}=$ $V_{k}^{\text {nom }}\left[\right.$ p.u.] is the nominal voltage magnitude of the $s$-th driver generator node, $\forall s \in \mathcal{I}_{n_{k}}^{p i n}$.

To achieve control objectives (10) and (11), for each non-pinner generator node $i \in \mathcal{I}_{k}^{\text {non-pin }}$, we design the following delayed consensus-based control strategy which weights both the voltage error with respect to the desired nominal value $V_{k, s}^{n o m}$ of the $s$-th driver generator node and the local voltage networked information as

$$
\begin{aligned}
u_{k, i}\left(t, \tau_{i j}^{k}(t), \tau_{i s}^{k}(t)\right)= & \gamma_{1} \sum_{j \in \mathcal{I}_{k}^{\text {lower }}} a_{i j}^{k}\left(V_{k, i}\left(t-\tau_{i j}^{k}(t)\right)-V_{k, j}\left(t-\tau_{i j}^{k}(t)\right)\right) \\
& +\gamma_{2} \sum_{s \in \mathcal{I}_{n_{k}}} b_{i s}^{k}\left(V_{k, i}\left(t-\tau_{i s}^{k}(t)\right)-V_{k, s}^{n o m}\left(t-\tau_{i s}^{k}(t)\right)\right)
\end{aligned}
$$

where $a_{i j}^{k}$ model the presence/absence of a communication link between the bus $j$ and the bus $i$; $b_{i s}^{k}$ is the leader-adjacency matrix element (i.e., $\mathcal{B}_{k}$, see Section 2.1$) ; \tau_{i j}^{k}(t)$ and $\tau_{i s}^{k}(t)$ are the communication latencies arising from the information exhange among the smart controllers; $\gamma_{1}$ and $\gamma_{2} \in \mathbb{R}$ are the control gains to be tuned.

Conversely, to fulfill the control objectives in (12) and (13), we propose for each capacitor bank $p \in \mathcal{I}_{N_{c}^{k}}$, the following delayed consensus-based controller:

$$
\begin{aligned}
u_{k, p}\left(t, \tau_{p j}^{k}(t), \tau_{p s}^{k}(t)\right)= & \delta_{1} \sum_{j \in \mathcal{I}_{k}^{\text {lower }}} a_{p j}^{k}\left(V_{k, p}\left(t-\tau_{p j}^{k}(t)\right)-V_{k, j}\left(t-\tau_{p j}^{k}(t)\right)\right) \\
& +\delta_{2} \sum_{s \in \mathcal{I}_{n_{k}}} b_{p s}^{k}\left(V_{k, p}\left(t-\tau_{p s}^{k}(t)\right)-V_{k, s}^{\text {nom }}\left(t-\tau_{p s}^{k}(t)\right)\right)
\end{aligned}
$$


where $a_{p j}^{k}$ models the communication topology emerging from the presence/absence of a communication link between the $p$-th and $j$-th bus, while $b_{p s}^{k}$ models the presence/absence of communication among bus $p$ and pinner bus $s ; \tau_{p j}^{k}(t)$ and $\tau_{p s}^{k}(t)$ are the heterogeneous time-varying delays; $\delta_{1}$ and $\delta_{2} \in \mathbb{R}$ are the control gains to be tuned in order to maintain the reactive power of the bus $p$ within its operating range $\left[Q_{k, p}^{\min }, Q_{k, p}^{\max }\right]$.

\section{Case Study}

In this section, we prove the effectiveness of the proposed cluster-oriented cooperative control strategy (based on the Driver Generator nodes Selection Algorithm in Section 3.1) in guaranteeing the voltage regulation of an MMGs system, despite the presence of communication time-varying delays and/or possible load variations. More specifically, we consider an MMGs system consisting of $k=2$ identical IEEE 14-bus test systems MGs sharing information via a double-layer communication network, as in Figure 2. Each MG consists of $N_{g^{1}}=N_{g^{2}}=5$ distributed generators, i.e., buses 1, 2, 3, 6, 8, and $N_{c^{1}}=N_{c^{2}}=9$ capacitor banks with twenty power transmission lines, respectively. Details about line impedances, load and reactive power limits were provided in [37], while initial conditions for the $N^{k}$ electrical bus within each $M G_{k}(k=1,2)$ were reported in Table 1 , where the rated voltage value $V^{\text {rated }}$ to be imposed to the whole MMGs system was also reported. Note that, the initial conditions for each electrical bus within $M G_{1}$ and $M G_{2}$ are randomly chosen according to their acceptable operative ranges.

Without loss of generality, we assume that, within each $M G_{k}$, the intra-cluster communication topology perfectly matches the physical one described by the power transmission lines. Indeed, since the electrical topology satisfies the global reachability property of generator nodes, the above assumption can be made. However, we remark that any other communication topology meeting the global reachability property of generator nodes can be adopted to prove the effectiveness and the robustness of our control strategies [11]. The inter-cluster communication topology $\tilde{\mathcal{G}}$ is instead determined via Algorithm 1 to guarantee a faster synchronization process of all the electrical buses to the voltage-rated value $V^{\text {rated }}$. Namely, by setting $n_{k}=3 \forall k=1,2$, the algorithm returns, for each $M G_{k}$, the optimal configuration for driver generator nodes corresponding to generator nodes 2, 6 and 8 with a communication topology described by the following Laplacian matrix:

$$
\tilde{\mathcal{L}}=\left[\begin{array}{cccccc}
3 & 0 & 0 & -1 & -1 & -1 \\
0 & 3 & 0 & -1 & -1 & -1 \\
0 & 0 & 3 & -1 & -1 & -1 \\
-1 & -1 & -1 & 3 & 0 & 0 \\
-1 & -1 & -1 & 0 & 3 & 0 \\
-1 & -1 & -1 & 0 & 0 & 3
\end{array}\right]
$$

The multiple time-varying delays, affecting both the inter-cluster and the intra-cluster communication networks, have been emulated as random variables with uniform distribution within the range $\left[0, \tau^{\star}\right]$, where $\tau^{\star}=0.1[s]$ is the upper bound setting above the typical average end-to-end communication delay in a wireless network $[11,38,39]$.

Moreover, the control gains parameters for the cooperative control strategies (9) (14) (15) are selected to avoid the reactive power exceeding its maximum/minimum allowable values. Their values are listed in Table 1.

Simulation analysis, carried out via the Matlab/Simulink (Release 2019a) platform, involves two operative scenarios: (i) Nominal scenario, where only heterogeneous time-varying delays are considered; (ii) Load Fluctuations scenario, where both multiple time-varying delays and load variations are taken into account. 


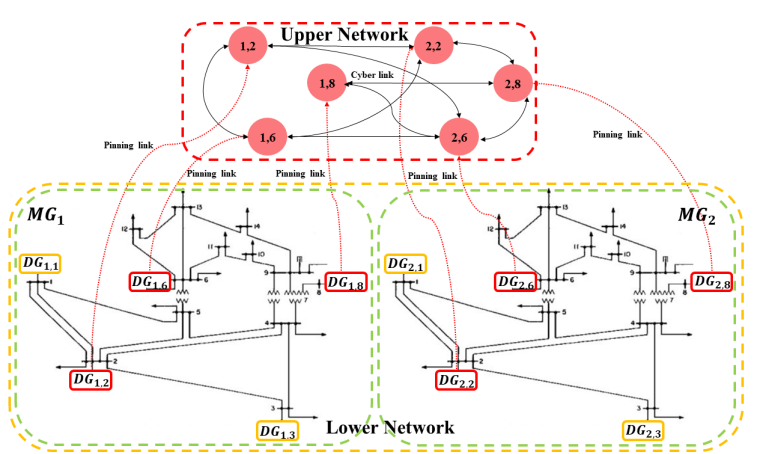

(a)

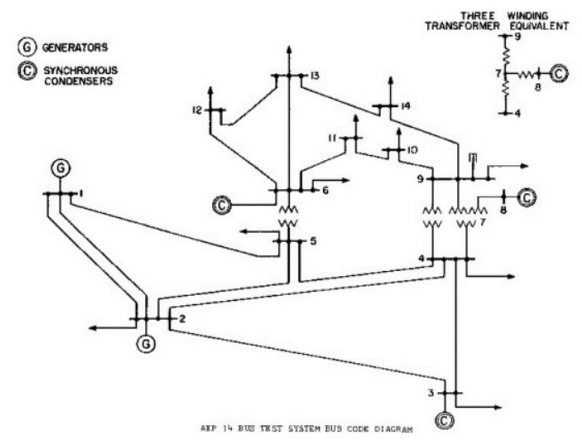

(b)

Figure 2. Multiple MicroGrids Modeling (MMGs) system composed of two identical IEEE14-bus Test systems: (a) Two-layer network: intra-cluster lower network shown by the yellow dotted line and the inter-cluster upper network shown by the red dotted line; (b) Single IEEE 14-bus test system.

Table 1. Two IEEE 14-bus test systems MMGs system: parameters values.

\begin{tabular}{cc}
\hline Initial Conditions for the Multiple MG Cluster System & \\
\hline Voltage magnitude of generator bus $\forall k=1,2 \quad[p . u]$. & $\begin{array}{r}V_{k, 1}(0)=1.06 ; V_{k, 2}(0)=0.97 ; V_{k, 5}(0)=1.01 ; \\
V_{k, 6}(0)=0.97 ; V_{k, 8}(0)=0.97\end{array}$ \\
\hline Cluster Voltage-rated value $V^{\text {rated }}[p . u]$. & $V^{\text {rated }}=1.03$ \\
\hline & $Q_{k, 4}(0)=-0.039 ;$ \\
$Q_{k, 5}(0)=0.016 ;$ \\
$Q_{k, 7}(0)=0.00 ;$ \\
Reactive power of capacitor bank bus $p \in \mathcal{I}_{N_{c}^{k}} \forall k=1,2 \quad[p . u]$. & $Q_{k, 9}(0)=0.166 ;$ \\
& $Q_{k, 10}(0)=0.058 ;$ \\
$Q_{k, 11}(0)=0.018 ;$ \\
$Q_{k, 12}(0)=0.016 ;$ \\
Control Gains & $Q_{k, 13}(0)=0.058 ;$ \\
Control gains $\gamma_{i}$ & $Q_{k, 14}(0)=0.05 ;$ \\
Control gains $\delta_{i}$ & $\gamma_{i}=-0.5 \forall i=1,2$ \\
& $\delta_{i}=200 \forall i=1,2$ \\
& $k_{Q}=1$ \\
\hline
\end{tabular}

\subsection{Nominal Scenario}

In this scenario, where no load variations are considered, the two MGs are electrically disconnected from each other at $t=0[\mathrm{~s}]$ and, then, connected at $t=5[\mathrm{~s}]$. Therefore, the lower controller and the upper controllers (as well as its corresponding pinning links) are disabled at $t=0[\mathrm{~s}]$ and switch-on at $t=5[s]$.

The results in Figure 3 confirm the effectiveness of the proposed control approach in guaranteeing the cluster-oriented double-layer cooperative control despite the presence of heterogeneous time-varying communication delays. Specifically, since upper-layer controllers $u_{k, s} \forall s \in \mathcal{I}_{n_{k}}^{p i n}, \forall k \in \mathcal{I}_{M}$ in (9) are inactive during the time interval $[0,5][s]$, generator voltage magnitudes do not reach the nominal value $V_{k}^{n o m}=1.03[p . u],. k=1,2$ (Figure 3a,b). When, at $t=5[s]$ the cluster-oriented cooperative control strategy is enabled, the voltage magnitudes of all the generator buses (including the driver generator nodes) within both the $M G_{1}$ and the $M G_{2}$ converge to the desired nominal values $V_{k}^{n o m}=1.03[p . u$.] $, k=1,2$ (see Figure $3 \mathrm{a}, \mathrm{b}$, respectively). As a result, the control objectives in (7) and (8) are fulfilled. As a consequence, the lower cooperative control protocol $u_{k, p} \forall p \in N_{c^{k}}, k=1,2$ in (15) drives the reactive power $Q_{k, p}$ [p.u.] of each capacitor bank $p$ to guarantee that the corresponding voltage magnitudes $V_{k, p}$ reach the nominal value $V_{k}^{\text {nom }}=1.03,[p . u$.$] , as shown in Figure 3c-e.$ Figure 4 highlights the time histories of reactive powers of capacitor banks within $M G_{1}$ and $M G_{2}$. 
Thus, the control goals in (10) and (11), as well as the control objectives in (12) and (13), are satisfied. Indeed, all the voltage values of the $N_{c}^{k}$ capacitor banks in both $M G_{1}$ and $M G_{2}$ converge to the voltage imposed by the $n_{k}$ pinner generators, i.e., $V_{k}^{n o m}=1.03[p . u$.]. In conclusion, the double-layer control architecture is able to regulate voltage values throughout the MGs cluster despite the presence of multiple time-varying communication delays.

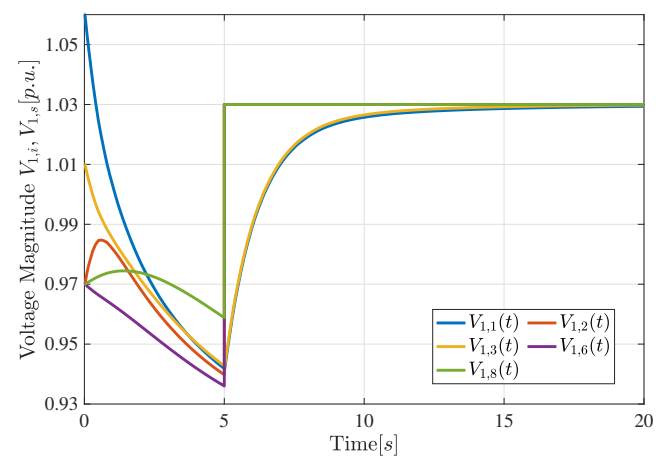

(a)

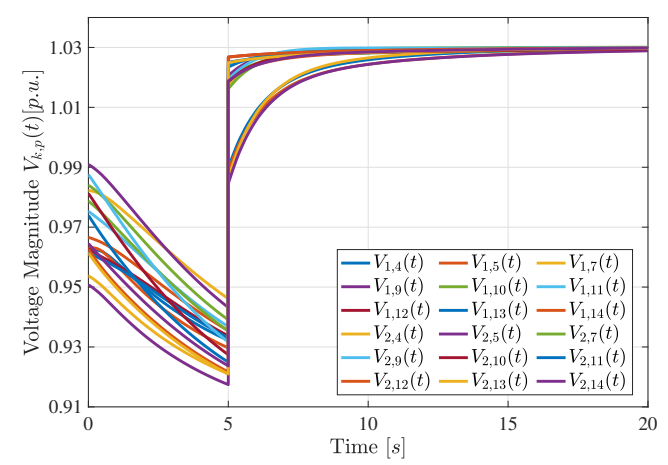

(c)

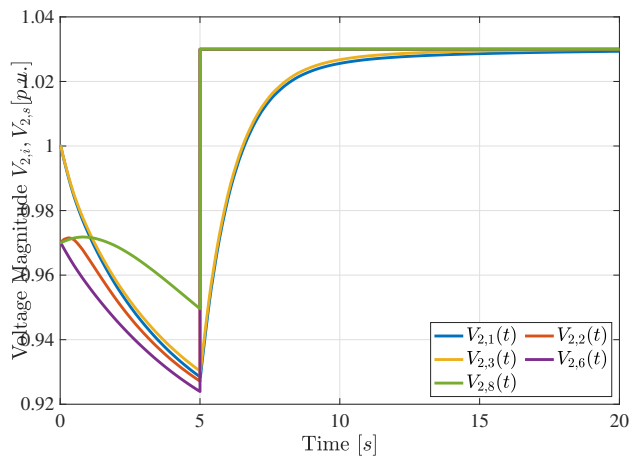

(b)

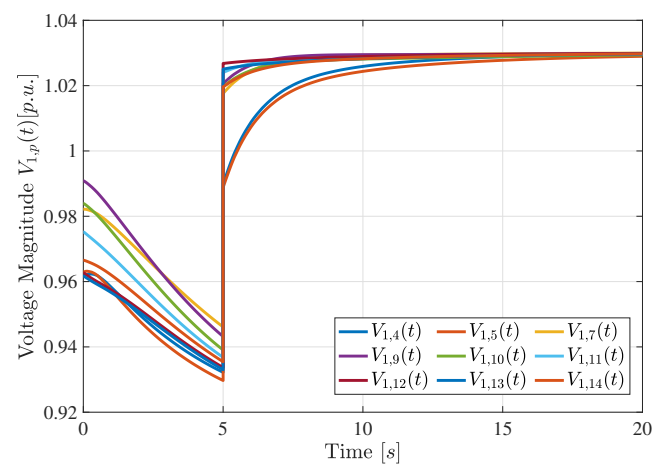

(d)

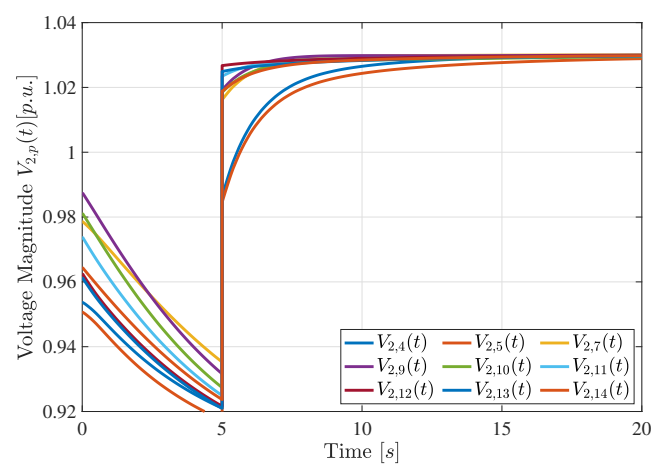

(e)

Figure 3. Cluster Oriented Double Layer Cooperative Control in the nominal scenario. Time history of: (a) $M G_{1}$ Non-Pinner Generator bus and Pinner Generator bus voltages $V_{1, i}, i \in N_{g}^{1}[p . u$.] and $V_{1, s}^{n o m}, \mathrm{~s}=2,6,8$; (b) $M G_{2}$ Non-Pinner Generator bus and Pinner Generator bus voltages $V_{2, i}, i \in$ $N_{g}^{2}[p . u$. $]$ and $V_{2, s}^{n o m}, s=2,6,8$; (c) MMGs Voltage Values $V_{k, p}, \forall k=1,2, p \in N_{c}^{k}$; (d) $M G_{1}$ Capacitor bank bus voltages $V_{1, p}, p \in N_{c}^{1}\left[p . u\right.$.]; (e) $M G_{2}$ Capacitor bank bus voltages $V_{2, p}, p \in N_{c}^{2}[p . u$.] 


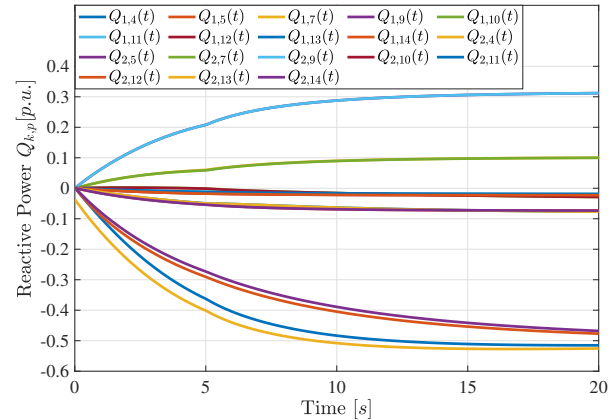

(a)

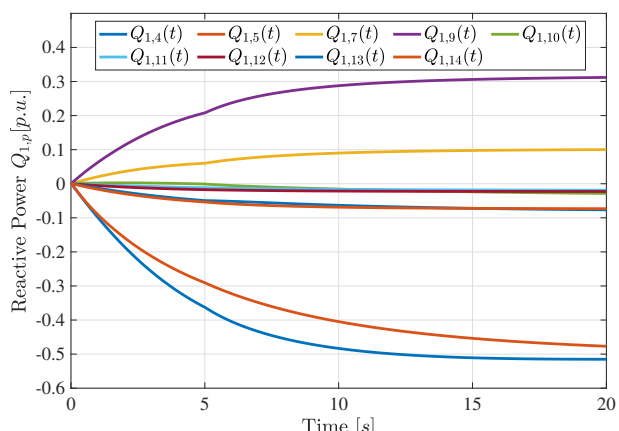

(b)

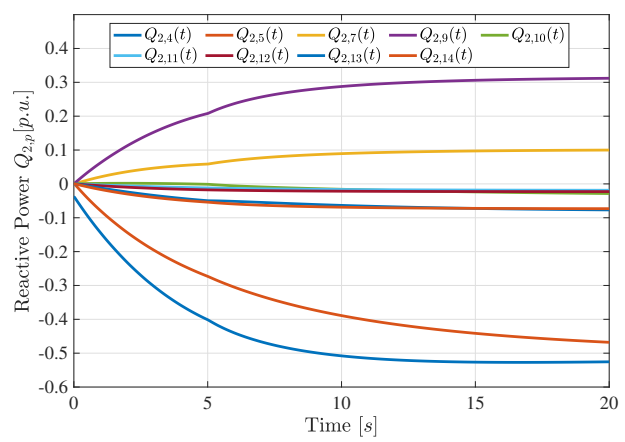

(c)

Figure 4. Cluster Oriented Double Layer Cooperative Control in Nominal scenario. Time history of: (a) Reactive power of the MMGs $Q_{k, p}, k=1,2, p \in N_{c}^{k}\left[p . u\right.$.]; (b) $M G_{1}$ Capacitor bank bus Reactive power $Q_{1, p}, p \in N_{c}^{1}\left[p . u\right.$.]; (c) $M G_{2}$ Capacitor bank bus Reactive power $Q_{2, p}, p \in N_{c}^{2}$ [p.u.].

\subsection{Load Fluctuations Scenario}

In this section, we present the results of the performance analysis of our cooperative controllers, confirming voltage regulation of the overall MMGs when load fluctuations occur. Indeed, since in a real practical situation, the load demand is sensitive to frequent changes, it is particularly desirable to evaluate the robustness of the proposed control strategies in this crucial scenario [1].

The appraised load fluctuations profile $L(t)$ is the one depicted in Figure 5a, where a maximum load variation of $\pm 50 \%$ can be observed.

Figure $5 b-f$ reveal the effectiveness and robustness of the proposed cluster-oriented, two-layer control protocol in counteracting sudden fluctuations of the load request, while achieving good voltage regulation performances despite the presence of heterogeneous time-varying delays. More specifically, when the upper cooperative controllers are not enabled from $[0 ; 5][s]$, all the generator buses' voltages do not converge to the nominal value $V_{k}^{\text {nom }}=1.03\left[p . u\right.$.] imposed to $M G_{k}, k \in \mathcal{I}_{M}$. However, when the cooperative control protocol is switched on at $t=5[s]$, the generator buses' voltages quickly converge to the nominal value despite the presence of both load variation and multiple time-varying delays (see Figure 5b,c). Accordingly, the voltage magnitudes of all buses within the MMGs system achieve the desired set point (see Figure 5d), thus proving the ability of the designed controllers to react to load fluctuations by inducing a reactive power variation (see Figure $6 \mathrm{a}-\mathrm{c}$ ) and promptly restore the voltage to the required level. Finally, Figure 5e,f show the time histories of the voltages of each bus $p \in N_{c^{k}} k=1,2$ in the $M G_{1}$ and the $M G_{2}$, respectively. In conclusion, all the control goals (10)-(13) are also fulfilled in this simulation scenario. 


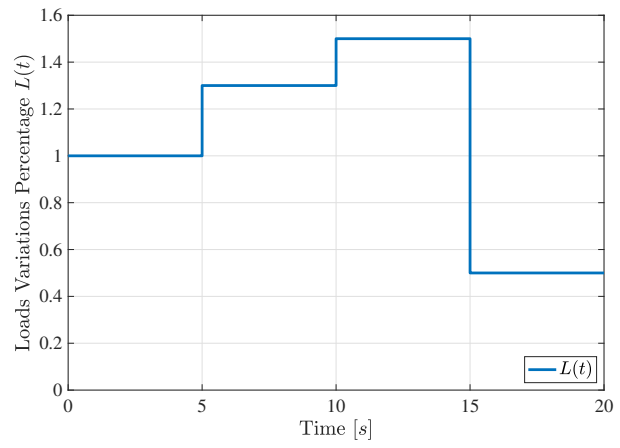

(a)

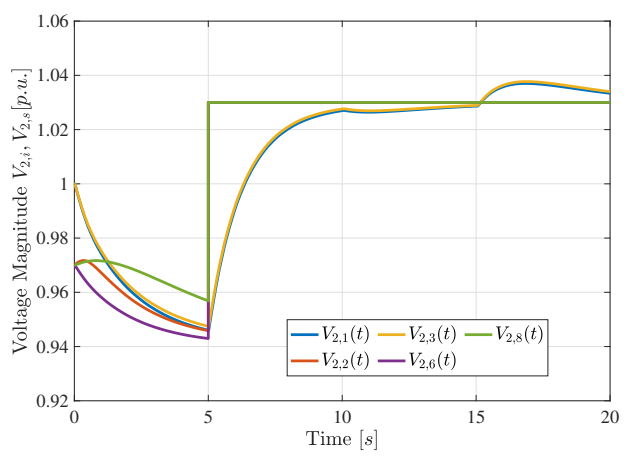

(c)

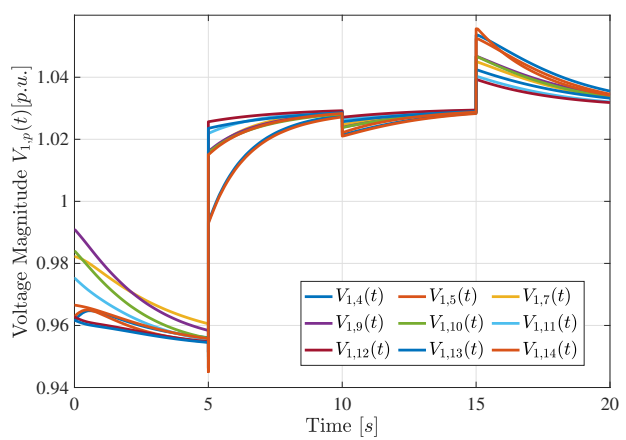

(e)

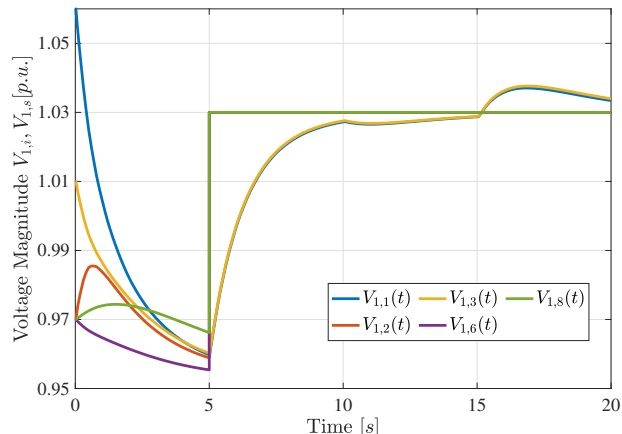

(b)

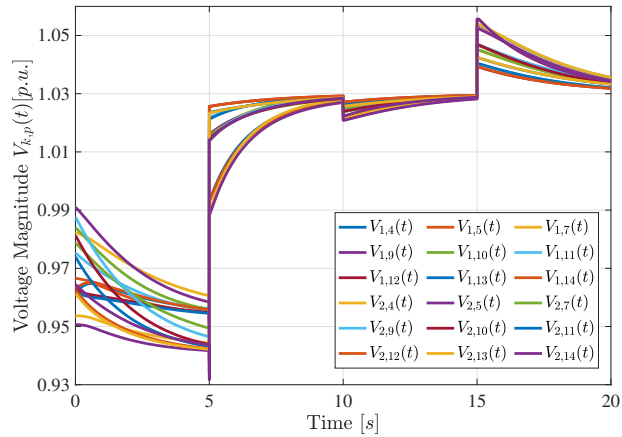

(d)

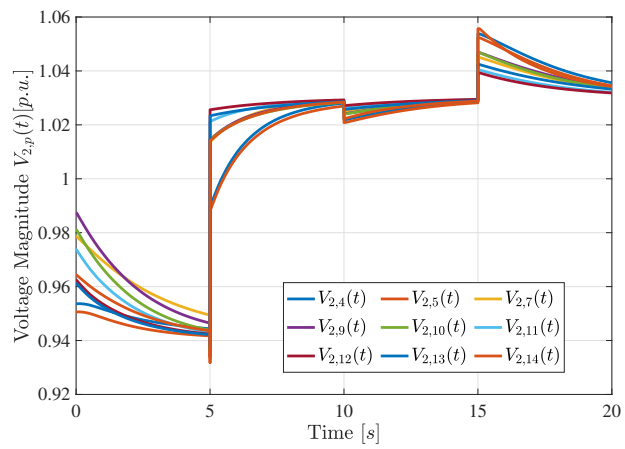

(f)

Figure 5. Cluster-Oriented Double-Layer Cooperative Control in Load Fluctuations scenario. Time history of: (a) Variable load profile $L(t)$. Percentage variation with respect to nominal value; (b) $M G_{1}$ Non-Pinner Generator bus and Pinner Generator bus voltages $V_{1, i}, i \in N_{g}^{1}\left[p . u\right.$.] and $V_{1, s}^{\text {nom }}, \mathrm{s}=2,6,8$; (c) $M G_{2}$ Non-Pinner Generator bus and Pinner Generator bus voltages $V_{2, i}, i \in N_{g}^{2}[p . u$.] and $V_{2, s}^{n o m}, \mathrm{~s}=2,6,8$; (d) MMGs Voltage Values $V_{k, p}, \forall k=1,2, p \in N_{c}^{k}$; (e) $M G_{1}$ Capacitor bank bus voltages $V_{1, p}, p \in N_{c}^{1}\left[p . u\right.$.] (f) $M G_{2}$ Capacitor bank bus voltages $V_{2, p}, p \in N_{c}^{2}[p . u$.]. 


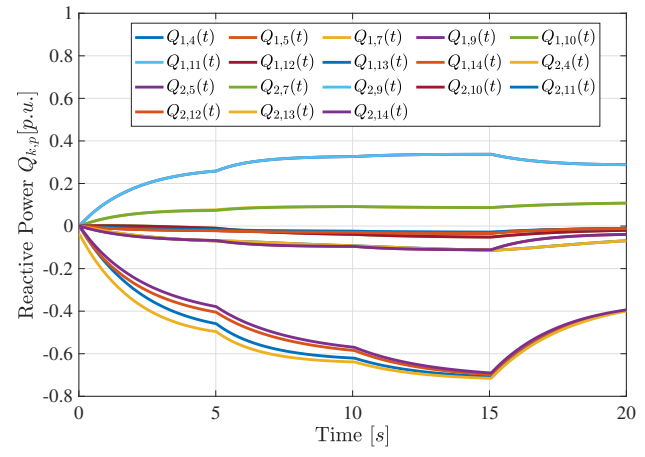

(a)

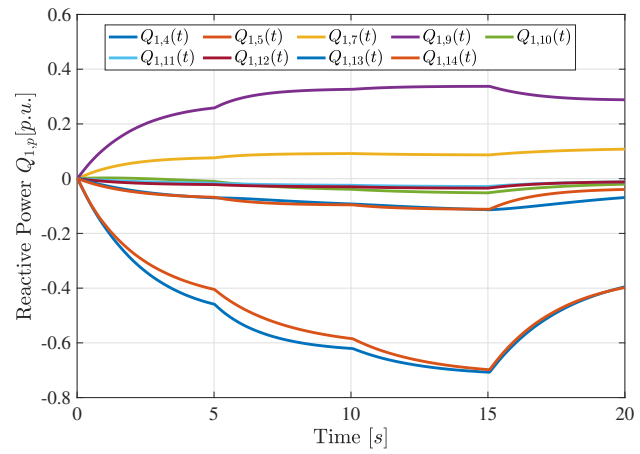

(b)

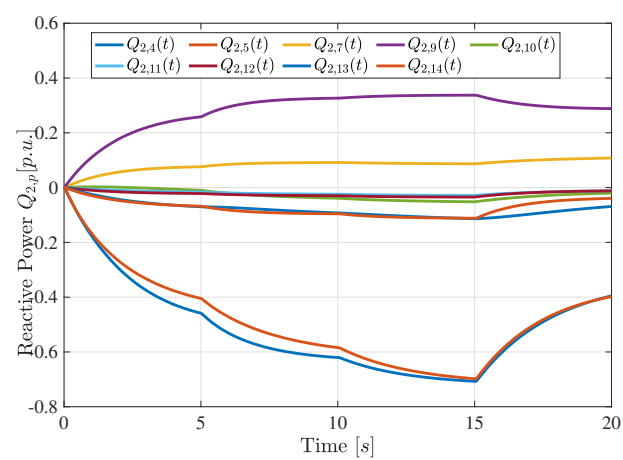

(c)

Figure 6. Cluster Oriented Double Layer Cooperative Control in Load Fluctuations scenario. Time history of: (a) Reactive power of the MMGs $Q_{k, p}, k=1,2, p \in N_{c}^{k}\left[p . u\right.$.]; (b) $M G_{1}$ Capacitor bank bus Reactive power $Q_{1, p}, p \in N_{c}^{1}$ [p.u.]; (c) $M G_{2}$ Capacitor bank bus Reactive power $Q_{2, p}, p \in N_{c}^{2}$ [p.u.].

\subsection{Comparison Analysis}

Here, we compare the performances of our fully delayed control strategies in (9), (14) and (15), which were designed according to delayed information processing with respect to the ones achievable with the following control strategy:

$$
\begin{aligned}
& u_{k, s}^{t}\left(t, V^{\text {rated }}, \tau_{l s}(t)\right)=V^{\text {rated }}+k_{Q}\left(\sum_{l \in \mathcal{I}^{p i n}} a_{s l}\left(Q_{k^{\prime}, l}\left(t-\tau_{l s}(t)\right)-Q_{k, s}(t)\right)\right), \\
& u_{k, i}^{t}\left(t, \tau_{i j}^{k}(t), \tau_{i s}^{k}(t)\right)=\gamma_{1} \sum_{j \in \mathcal{I}_{k}^{\text {lower }}} a_{i j}^{k}\left(V_{k, i}(t)-V_{k, j}\left(t-\tau_{i j}^{k}(t)\right)\right)+\gamma_{2} \sum_{s \in \mathcal{I}_{n_{k}}} b_{i s}^{k}\left(V_{k, i}(t)-V_{k, s}^{\text {nom }}\left(t-\tau_{i s}^{k}(t)\right)\right), \\
& \left.u_{k, p}^{t}\left(t, \tau_{p j}^{k}(t), \tau_{p s}^{k}(t)\right)=\delta_{1} \sum_{j \in \mathcal{I}_{k}^{\text {lower }}} a_{p j}^{k}\left(V_{k, p}(t)\right)-V_{k, j}\left(t-\tau_{p j}^{k}(t)\right)\right)+\delta_{2} \sum_{s \in \mathcal{I}_{n_{k}}} b_{p s}^{k}\left(V_{k, p}(t)-V_{k, s}^{n o m}\left(t-\tau_{p s}^{k}(t)\right)\right) .
\end{aligned}
$$

which only involves the transmission delays of the information received from neighbors. Therefore, each controller in (17a)-(17c) consists of two different terms-a local action depending on the state variables of the generic node itself (measured onboard) without any delay, and an action depending on the information received from neighboring nodes (distributed generators and/or capacitor banks) through the communication network that was affected by a time-varying communication delay. Exemplar results, reported in Figure 7, refer to the nominal scenario detailed in Section 4.1. 


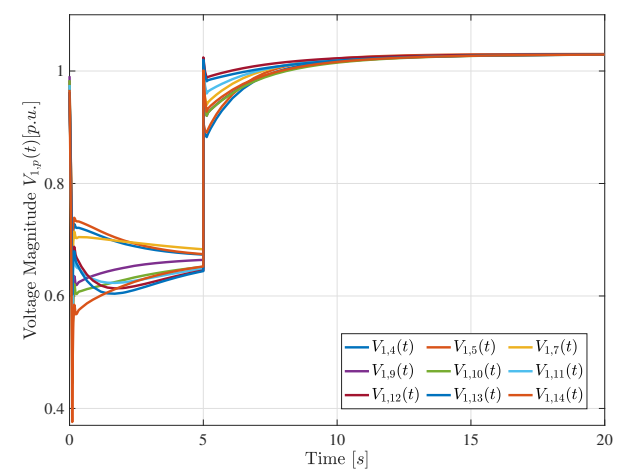

(a)

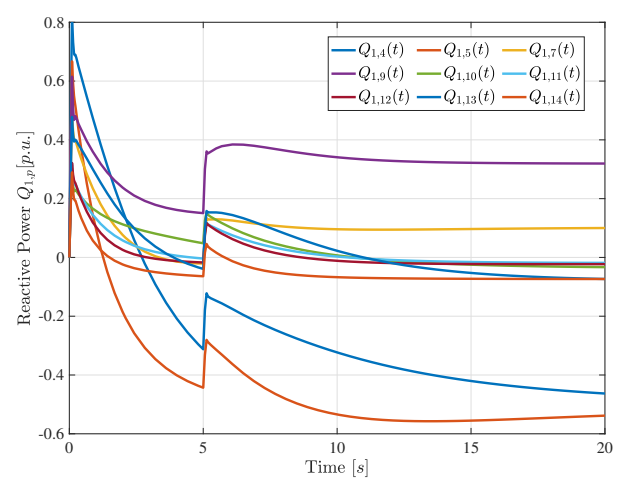

(c)

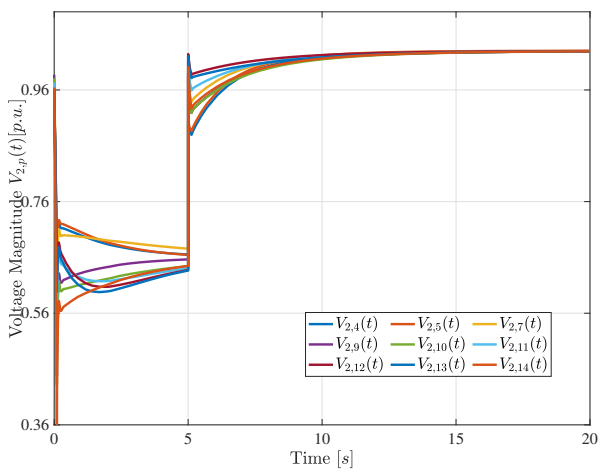

(b)

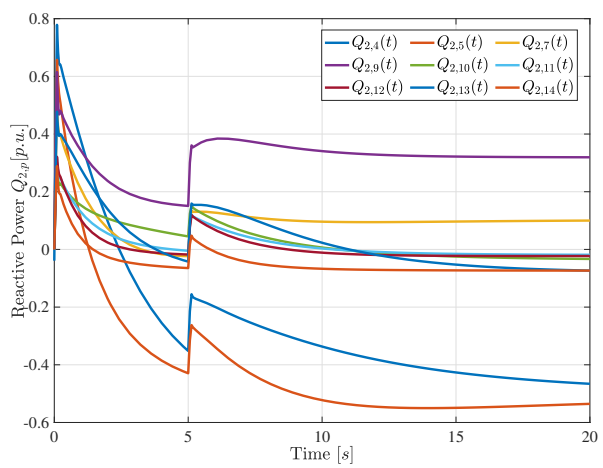

(d)

Figure 7. Comparison analysis of the proposed fully delayed Cluster Oriented Double Layer Cooperative Control with respect to the partial delayed control protocols in (17a)-(17c) involving only transmission delays in the nominal scenario. Time history of: (a) Voltage Values $V_{1, p}, p \in N_{c}^{1}$, (b) Voltage Values $V_{2, p}, p \in N_{c}^{2}$, (c) Reactive power of $M G_{1}, Q_{1, p}, p \in N_{c}^{1}$, (d) Reactive power of $M G_{2}, Q_{2, p}, p \in N_{c}^{2}$.

As it is possible to observe, the proposed fully delayed Cluster-Oriented Double-Layer Cooperative Control approach achieves better leader tracking performance in terms of voltages and reactive power with respect to the results obtained via controllers in (17a)-(17c) (depicted in Figure 7). This is due to the benefit introduced by networked-induced delays on the overall MMGs system $[40,41]$ which improves the closed-loop performances. Hence, the advantages obtained by considering random delays and their effects on the closed-loop network in the control design phase have been shown. Therefore, by implementing our distributed control strategies via fully outdated information, any possible instability sources can be counteracted $[42,43]$.

\section{Conclusions}

In this paper, the voltage regulation problem, as well as the optimal economical power sharing, for a multiple MGs cluster system in the presence of heterogeneous time-varying communication latency has been investigated and solved via a two-layer distributed control approach, which also leverages the MSF method. The proposed control architecture, based on cluster-oriented distributed cooperative control strategies, is able to guarantee an optimal energy management of the overall MMGs system despite the presence of heterogeneous time-varying communication delays. The numerical analysis was performed on the realistic case study of an MMGs system consisting of two IEEE 14-bus test systems. Simulation results, both in nominal and load fluctuations scenarios, have disclosed the effectiveness and robustness of the proposed control architecture. 
Author Contributions: A.A., B.C., A.P., S.S. and A.V.: Conceptualization, methodology, data curation. All authors have read and agreed to the published version of the manuscript.

Funding: This research received no external funding.

Conflicts of Interest: The authors declare no conflict of interest.

\section{Nomenclature}

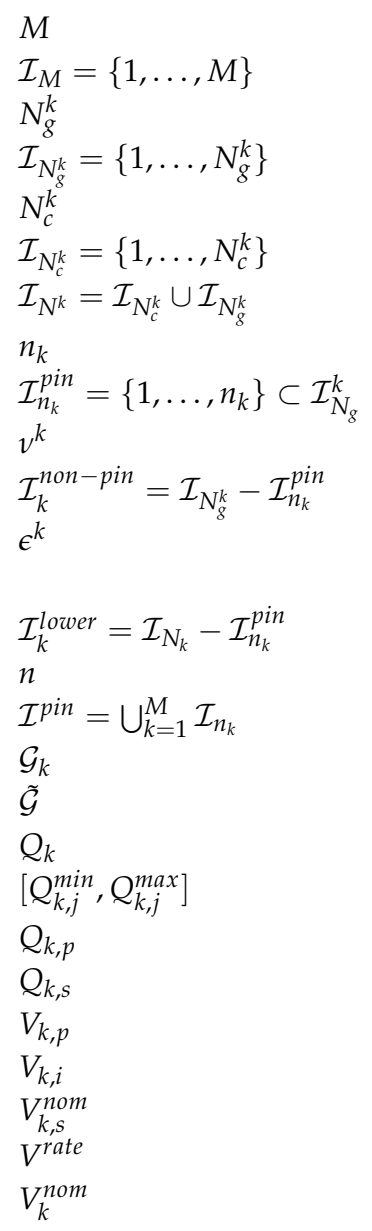

The number of MGs within the MMGs

The set of MGs within the MMGs

Number of distributed generators within the $k$-th MG

The set of distributed generators within the $k$-th MG

Number of capacitor banks within the $k$-th MG

The set of capacitor banks within the $k$-th MG

The set of total buses within the $k$-th MG

The number of driver generator nodes within the $k$-th MG

The set of driver generator nodes within the $k$-th MG

The number of non-pinner generator nodes within the $k$-th MG

The set of the $v^{k}$ non-pinner generators nodes within the $k$-th MG

The number of non-pinner generator nodes plus the $N_{c}^{k}$ capacitor bank within the $k$-th MG

The set of $\epsilon^{k}$ nodes within $k$-MG, involved into the lower cyber layer

The number of total driver generator nodes within MMGs

The set of all $n$ driver generator nodes within the MMGs

The intra-cluster cyber network of the $k$-th MG (except for driver generator nodes)

The inter-cluster cyber network

Output Reactive power of the $k$-th MG

Reactive Power Operating Range of the $j$-th electrical bus within $k$-th MG

Reactive power of the $p$-th capacitor bank within $k$-th MG

Reactive power of the $s$-th pinner generator within $k$-th MG

Voltage magnitude of the $p$-th capacitor bank within $k$-th MG

Voltage magnitude of the $i$-th non pinner generator node within $k$-th MG

Voltage magnitude of the $s$-th pinner generator node within $k$-th MG

Rated voltage magnitude imposed on the MMGs

Nominal Voltage magnitude imposed on the $k$-th MG

\section{References}

1. Andreotti, A.; Caiazzo, B.; Petrillo, A.; Santini, S.; Vaccaro, A. Robust Finite-time Voltage Restoration in Inverter-Based Microgrids via Distributed Cooperative Control in presence of communication time-varying delays. In Proceedings of the 2020 IEEE International Conference on Environment and Electrical Engineering and 2020 IEEE Industrial and Commercial Power Systems Europe (EEEIC/I\&CPS Europe), Web Conference, Madrid, Spain, 9-12 June 2020; pp. 1-6.

2. Sen, S.; Kumar, V. Microgrid control: A comprehensive survey. Annu. Rev. Control 2018, 45, 118-151. [CrossRef]

3. Sedhom, B.E.; El-Saadawi, M.M.; Hatata, A.Y.; Abd-Raboh, E.H.E. H-Infinity versus model predictive control methods for seamless transition between islanded-and grid-connected modes of microgrids. IET Renew. Power Gener. 2019, 14, 856-870. [CrossRef]

4. Mumtaz, F.; Bayram, I.S. Planning, operation, and protection of microgrids: An overview. Energy Procedia 2017, 107, 94-100. [CrossRef]

5. Badal, F.R.; Das, P.; Sarker, S.K.; Das, S.K. A survey on control issues in renewable energy integration and microgrid. Prot. Control Mod. Power Syst. 2019, 4, 8. [CrossRef]

6. Mahmood, H.; Michaelson, D.; Jiang, J. Accurate reactive power sharing in an islanded microgrid using adaptive virtual impedances. IEEE Trans. Power Electron. 2014, 30, 1605-1617. [CrossRef] 
7. De Azevedo, R.; Cintuglu, M.H.; Ma, T.; Mohammed, O.A. Multiagent-based optimal microgrid control using fully distributed diffusion strategy. IEEE Trans. Smart Grid 2017, 8, 1997-2008. [CrossRef]

8. Andreotti, A.; Petrillo, A.; Santini, S.; Vaccaro, A.; Villacci, D. A decentralized architecture based on cooperative dynamic agents for online voltage regulation in smart grids. Energies 2019, 12, 1386. [CrossRef]

9. Fan, Y.; Zhang, C.; Song, C. Sampling-based self-triggered coordination control for multi-agent systems with application to distributed generators. Int. J. Syst. Sci. 2018, 49, 3048-3062. [CrossRef]

10. Yang, R.; Zhang, H.; Feng, G.; Yan, H.; Wang, Z. Robust cooperative output regulation of multi-agent systems via adaptive event-triggered control. Automatica 2019, 102, 129-136. [CrossRef]

11. Andreotti, A.; Caiazzo, B.; Petrillo, A.; Santini, S.; Vaccaro, A. Decentralized Smart Grid Voltage Control by Synchronization of Linear Multiagent Systems in the Presence of Time-Varying Latencies. Electronics 2019, 8, 1470. [CrossRef]

12. Lu, X.; Yu, X.; Lai, J.; Guerrero, J.M.; Zhou, H. Distributed secondary voltage and frequency control for islanded microgrids with uncertain communication links. IEEE Trans. Ind. Inform. 2016, 13, 448-460. [CrossRef]

13. Gong, P.; Lu, Z.; Lin, J.; Lv, Z.; Hu, L. Distributed secondary control based on cluster consensus of inhibitory coupling with power limit for isolated multi-microgrid. IET Gener. Transm. Distrib. 2019, 13, 4114-4122. [CrossRef]

14. Robbins, B.A.; Hadjicostis, C.N.; Domínguez-García, A.D. A two-stage distributed architecture for voltage control in power distribution systems. IEEE Trans. Power Syst. 2012, 28, 1470-1482. [CrossRef]

15. Zhao, B.; Wang, X.; Lin, D.; Calvin, M.M.; Morgan, J.C.; Qin, R.; Wang, C. Energy management of multiple microgrids based on a system of systems architecture. IEEE Trans. Power Syst. 2018, 33, 6410-6421. [CrossRef]

16. He, M.; Giesselmann, M. Reliability-constrained self-organization and energy management towards a resilient microgrid cluster. In Proceedings of the 2015 IEEE Power \& Energy Society Innovative Smart Grid Technologies Conference (ISGT), Washington, DC, USA, 18-20 February 2015; pp. 1-5.

17. Wu, X.; Xu, Y.; Wu, X.; He, J.; Guerrero, J.M.; Liu, C.C.; Schneider, K.P.; Ton, D.T. A two-layer distributed control method for islanded networked microgrid systems. arXiv 2018, arXiv:1810.08367

18. Che, L.; Zhang, X.; Shahidehpour, M.; Alabdulwahab, A.; Abusorrah, A. Optimal interconnection planning of community microgrids with renewable energy sources. IEEE Trans. Smart Grid 2015, 8, 1054-1063. [CrossRef]

19. Erol-Kantarci, M.; Kantarci, B.; Mouftah, H.T. Reliable overlay topology design for the smart microgrid network. IEEE Netw. 2011, 25, 38-43. [CrossRef]

20. Kumar, G.; Sattianadan, D.; Vijayakumar, K. A survey on power management strategies of hybrid energy systems in microgrid. Int. J. Electr. Comput. Eng. (2088-8708) 2020, 10, 1667.

21. Barra, P.; Coury, D.; Fernandes, R. A survey on adaptive protection of microgrids and distribution systems with distributed generators. Renew. Sustain. Energy Rev. 2020, 118, 109524. [CrossRef]

22. Lu, X.; Lai, J.; Yu, X.; Wang, Y.; Guerrero, J.M. Distributed coordination of islanded microgrid clusters using a two-layer intermittent communication network. IEEE Trans. Ind. Inform. 2017, 14, 3956-3969. [CrossRef]

23. Cortes, C.A.; Contreras, S.F.; Shahidehpour, M. Microgrid topology planning for enhancing the reliability of active distribution networks. IEEE Trans. Smart Grid 2017, 9, 6369-6377. [CrossRef]

24. Ajoulabadi, A.; Ravadanegh, S.N.; Mohammadi-Ivatloo, B. Flexible scheduling of reconfigurable microgrid-based distribution networks considering demand response program. Energy 2020, 196, 117024. [CrossRef]

25. Han, Y.; Zhang, K.; Li, H.; Coelho, E.A.A.; Guerrero, J.M. MAS-based distributed coordinated control and optimization in microgrid and microgrid clusters: A comprehensive overview. IEEE Trans. Power Electron. 2017, 33, 6488-6508. [CrossRef]

26. Qu, L.; Zhang, D.; Bao, Z. Active output-voltage-sharing control scheme for input series output series connected DC-DC converters based on a master slave structure. IEEE Trans. Power Electron. 2016, 32, 6638-6651. [CrossRef]

27. Wang, Y.; Nguyen, T.L.; Xu, Y.; Tran, Q.T.; Caire, R. Peer-to-Peer Control for Networked Microgrids: Multi-Layer and Multi-Agent Architecture Design. IEEE Trans. Smart Grid 2020. [CrossRef]

28. Lai, J.; Lu, X.; Yu, X.; Monti, A. Cluster-oriented distributed cooperative control for multiple AC microgrids. IEEE Trans. Ind. Inform. 2019, 15, 5906-5918. [CrossRef] 
29. Couto, M.; Lopes, J.P.; Moreira, C. Control strategies for Multi-Microgrids islanding operation through Smart Transformers. Electr. Power Syst. Res. 2019, 174, 105866. [CrossRef]

30. Lu, X.; Lai, J.; Yu, X. A Novel Secondary Power Management Strategy for Multiple AC Microgrids with Cluster-Oriented Two-Layer Cooperative Framework. IEEE Trans. Ind. Inform. 2020. [CrossRef]

31. Pecora, L.M.; Carroll, T.L. Master stability functions for synchronized coupled systems. Phys. Rev. Lett. 1998, 80, 2109. [CrossRef]

32. Jalili, M.; Sichani, O.A.; Yu, X. Optimal pinning controllability of complex networks: Dependence on network structure. Phys. Rev. E 2015, 91, 012803. [CrossRef]

33. Su, H.; Wang, X. Pinning Control of Complex Networked Systems: Synchronization, Consensus and Flocking of Networked Systems via Pinning; Springer: Berlin/Heisenberg, Germany, 2013.

34. Amani, A.M.; Jalili, M.; Yu, X.; Stone, L. Finding the most influential nodes in pinning controllability of complex networks. IEEE Trans. Circuits Syst. II: Express Briefs 2017, 64, 685-689. [CrossRef]

35. Fridman, E. Introduction to Time-Delay Systems: Analysis and Control; Springer: Berlin/Heisenberg, Germany, 2014.

36. Hase, Y. Handbook of Power System Engineering; John Wiley \& Sons: Hoboken, NJ, USA, 2007.

37. Zimmerman, R.D.; Murillo-Sánchez, C.E. MATPOWER 6.0 user's manual. Power Syst. Eng. Res. Cent. 2016.

38. Fiengo, G.; Lui, D.G.; Petrillo, A.; Santini, S. Distributed leader-tracking adaptive control for high-order nonlinear Lipschitz multi-agent systems with multiple time-varying communication delays. Int. J. Control 2019, 2, 1-13. [CrossRef]

39. Horalek, J.; Svoboda, T.; Holik, F. Analysis of the wireless communication latency and its dependency on a data size. In Proceedings of the 2016 IEEE 17th International Symposium on Computational Intelligence and Informatics (CINTI), Budapest, Hungary, 17-19 November 2016; pp. 000145-000150.

40. Zhang, X.M.; Han, Q.L.; Yu, X. Survey on recent advances in networked control systems. IEEE Trans. Ind. Inform. 2015, 12, 1740-1752. [CrossRef]

41. Fiengo, G.; Lui, D.G.; Petrillo, A.; Santini, S. Distributed robust output consensus for linear multi-agent systems with input time-varying delays and parameter uncertainties. IET Control Theory Appl. 2018, 13, 203-212. [CrossRef]

42. Petrillo, A.; Salvi, A.; Santini, S.; Valente, A.S. Adaptive synchronization of linear multi-agent systems with time-varying multiple delays. J. Frankl. Inst. 2017, 354, 8586-8605. [CrossRef]

43. Petrillo, A.; Pescape, A.; Santini, S. A secure adaptive control for cooperative driving of autonomous connected vehicles in the presence of heterogeneous communication delays and cyberattacks. IEEE Trans. Cybern. 2020. [CrossRef]

Publisher's Note: MDPI stays neutral with regard to jurisdictional claims in published maps and institutional affiliations.

(C) 2020 by the authors. Licensee MDPI, Basel, Switzerland. This article is an open access article distributed under the terms and conditions of the Creative Commons Attribution (CC BY) license (http:/ / creativecommons.org/licenses/by/4.0/). 